\title{
Elective Circulatory Arrest with Continuous Coronary Perfusion During Neurosurgical Operations
}

\author{
P. H. DAVISON*, D. J. S. McILVEEN, J. M. SMALL, AND V. CAMPKIN \\ From Midland Centre for Neurosurgery and Neurology, Smethwick, Staffordshire
}

The provision of a bloodless field during operations on certain intracranial aneurysms is highly desirable, and in a previous communication (Small et al., 1966) we described a method of elective circulatory arrest by artificial pacing of the heart at very rapid rates with an endocardial pacemaking electrode. It was shown that in the anaesthetized patient under moderate hypothermia $\left(31-32^{\circ} \mathrm{C}\right.$.) the blood pressure and cardiac output were very sensitive to changes in heart rate, and that circulatory arrest could be induced by rates as low as 180-200 a minute. This technique was successfully employed to arrest the circulation in 12 patients while they were being operated on for berry aneurysms. Unfortunately it proved impossible to retain control of the cardiac rhythm for more than 1 to 2 minutes, for the profound hypotension precipitated ventricular fibrillation and necessitated resuscitation with external cardiac massage and direct current shock. The need for resuscitation reduced the time during which elective arrest could be safely maintained with due regard to the oxygen requirements of the brain, and also increased the hazards and anxieties of the procedure. To overcome these difficulties the following method has been developed.

\section{METHOD}

The patient is anaesthetized with halothane and oxygen, and body temperature is reduced to $31-32^{\circ} \mathrm{C}$. (nasopharyngeal or mid-oesophageal) by surface cooling. Oscilloscopic electrocardiographic monitoring is maintained throughout. The patient is then transferred to the $X$-ray Department where a bipolar electrode catheter is placed in the apex of the right ventricle through a superficial right arm vein so that the rhythm can be captured at 1 volt.

Received March 15, 1968.

* Present address : Queen Elizabeth Hospital, Birmingham, 15.
A No. 13 Mahajan-Cliffton triple lumen catheter is modified in the following way: the proximal and distal balloons are removed and a single balloon made of seamless $0.6 \mathrm{~cm}$. ( $\frac{1}{4} \mathrm{in}$.) latex tubing having a capacity of approximately $60 \mathrm{ml}$. is tied on the central lumen $6 \mathrm{~cm}$. long and starting $6 \mathrm{~cm}$. from the tip. The catheter is moulded so that it will pass round the aortic arch. It is introduced into the aorta by a direct right femoral arteriotomy, and advanced until the tip is approximately 3.8 $\mathrm{cm}$. ( $1 \frac{1}{2} \mathrm{in}$.) above the aortic valve, so that distension of the balloon will occlude the aorta just proximate to the innominate artery. The position and behaviour of the catheter is checked by distending the balloon with radiopaque dye under fluoroscopic control (Fig. 1a and 1b). Another catheter introduced through a peripheral vein is positioned with its tip in the right atrium or pulmonary artery to monitor right heart pressures. The free lumens of the aortic catheter are used to monitor arterial pressure proximal and distal to the balloon.

Defibrillating shoulder-blade electrodes are strapped to the chest allowing DC shock to be applied immediately if ventricular fibrillation develops.

After placement of the catheters, the patient is transferred to the operating theatre, and the neurosurgical procedure begins. When circulatory arrest is required, the heart is paced artificially at $120-160$ beats a minute. The ascending aortic pressure falls, and when it has reached $20 \mathrm{~mm}$. $\mathrm{Hg}$ the balloon is rapidly inflated with $50 \mathrm{ml}$. saline to block the ascending aorta, the whole operation taking approximately 15 seconds. The pacemaker rate is then reduced until the ascending aortic pressure has risen to $50-100 \mathrm{~mm}$. $\mathrm{Hg}$ (Fig. 2). The initial pacing rate is $80-100$ and this is adjusted continually to maintain pressures within this range, thus ensuring continuous coronary perfusion during the arrest. To re-establish the general circulation, the pacing rate is transiently increased while the balloon is deflated, to avoid undue displacement of the catheter (Fig. 3).

With continuous coronary perfusion, control of the cardiac rhythm is not normally lost, but if ventricular fibrillation does occur during the arrest a DC shock is immediately administered through the shoulder-blade electrodes without earlier massage, and only momentary interruption of the surgical procedure is necessary. 


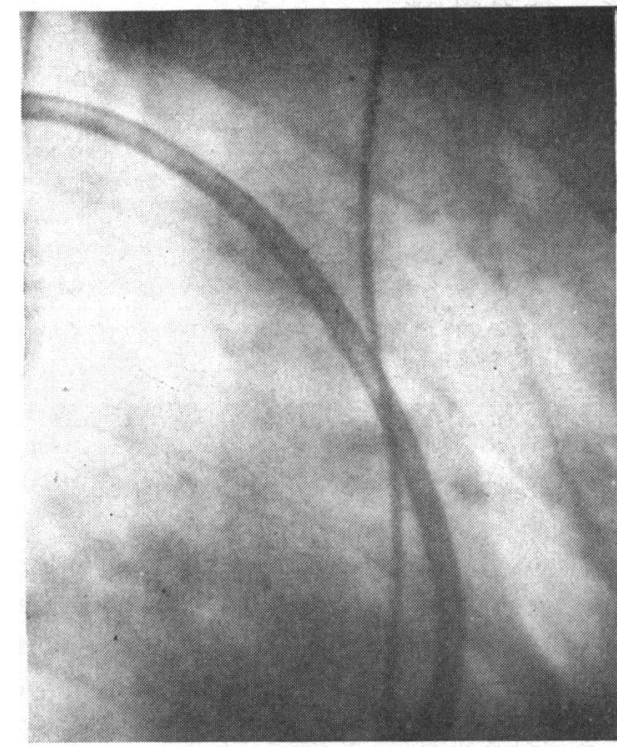

A

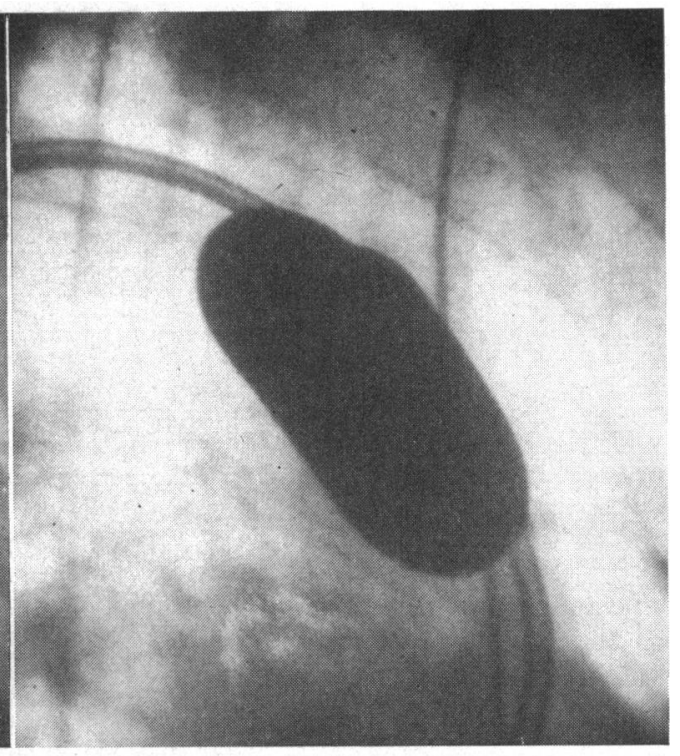

B

FIG. 1.-(A) Catheter in position in ascending aorta. (B) With balloon inflated.

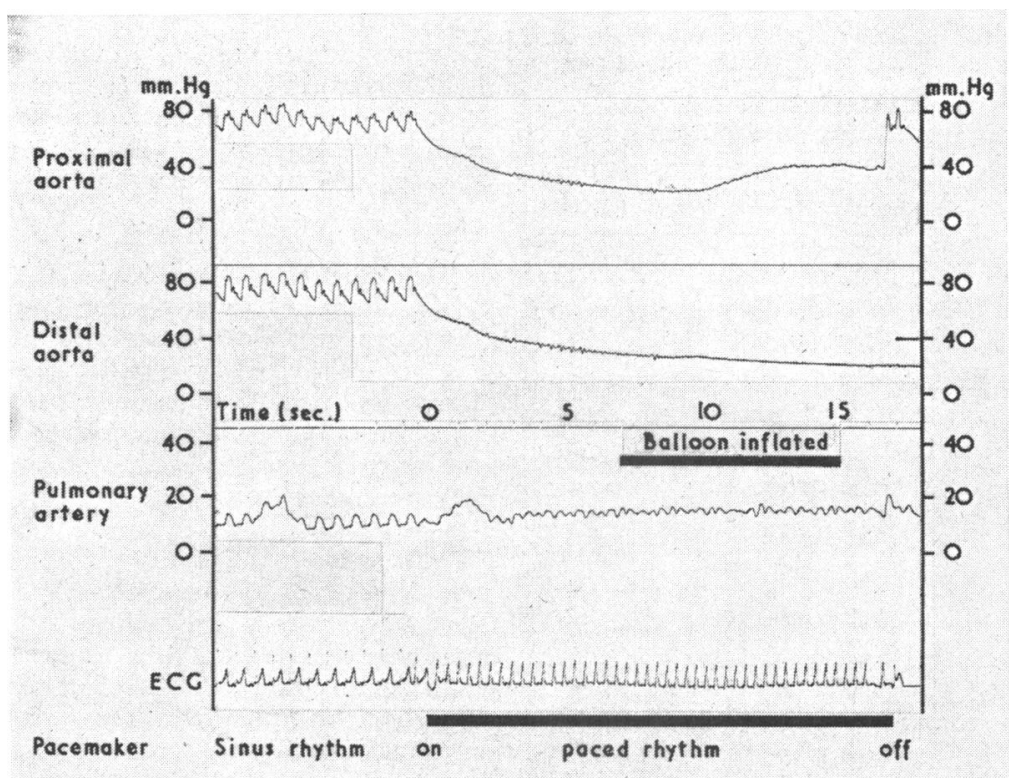

FIG. 2.-Start of circulatory arrest, showing timing of the balloon inflation. Note the return of proximal aortic pressure to normal.

Anaesthesia is discontinued during the arrest. 50 $100 \mathrm{mEq} 8$ per cent sodium bicarbonate are infused intravenously while the arrest is proceeding. Halothane and propranolol are used to reduce the pre-arrest blood pressure to $50-70 \mathrm{~mm}$. $\mathrm{Hg}$ systolic, and a further dose of propranolol may be given after arrest if the cardiac rhythm is disturbed.

The balloon catheter is removed immediately the need for arrest has passed and the femoral artery sutured after removal of all proximal and distal clot. 


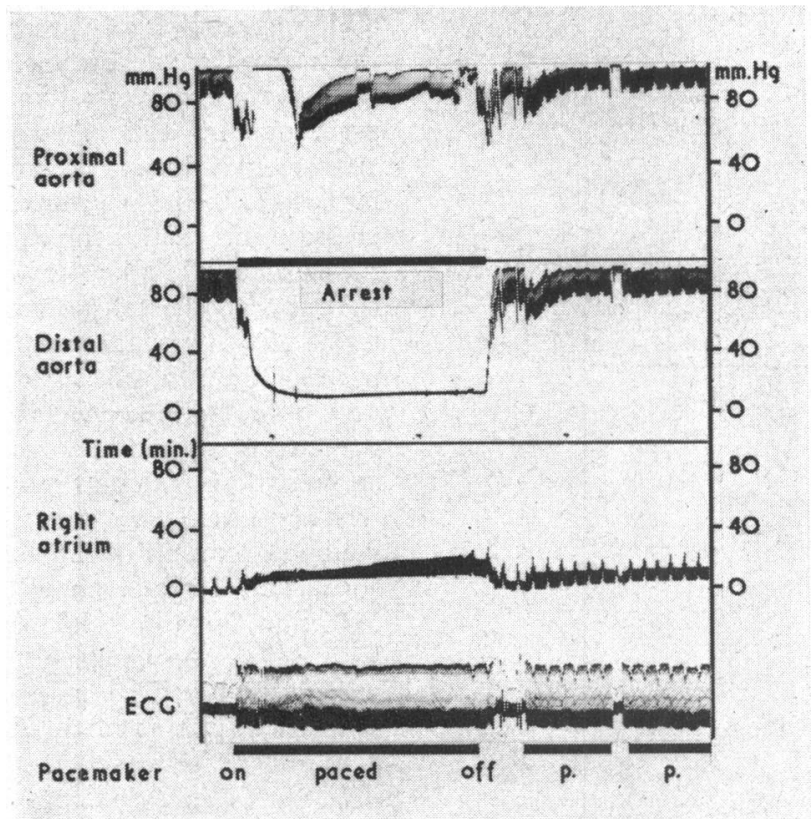

Fig. 3.-Elective arrest for approximately $1 \frac{1}{2}$ minutes. Note progressive rise in right atrial pressure during this period.

\section{Results}

This method has been used to produce circulatory arrest in 30 patients ( 12 male and 18 female) on 37 occasions, whose ages ranged from 12 to 62 years. All were suffering from cerebral vascular abnormalities requiring surgery for haemorrhage. The total duration of the circulatory arrest ranged from 50 seconds to $6 \frac{1}{2}$ minutes. In 7 patients two periods of arrest were necessary, and in these the combined duration ranged from 2 minutes 50 seconds to 9 minutes. There was an interval of 10 to 15 minutes between the two periods. In every case circulatory arrest was initiated at the request of the neurosurgeon to facilitate his operation in its critical phase. In addition to this, short periods of arrest not exceeding 30 seconds were usually provoked in each case at the beginning of the operation to ensure that the technique was working satisfactorily.

\section{INTERPRETATIONS}

The success of the method depends upon the capacity to regulate cardiac output by variation of the heart rate with the artificial pacemaker. The following changes have been constantly observed.

(1) Sudden onset of a rapid pacing stimulus is followed by a steep fall in right and left ventricular systolic pressures, with a simultaneous rise in cen- tral venous and right ventricular end-diastolic pressures (Fig. 4 and 5).

(2) The first normal sinus beat after a period of rapid pacing shows an abnormally high right atrial and right ventricular systolic pressure wave and an increased systemic pulse pressure indicative of supranormal filling and ejection (Fig. 5).

The depressions of cardiac function that accompany ventricular endocardial pacing may be due to a variety of causes. Loss of atrial systole will disturb the normal atrial transport mechanism. The abnormal origin and aberrant pathway of the pacing stimulus will directly reduce the efficiency of ventricular contraction, and indirectly reduce it by provoking a functional incompetence of the $A V$ valves, and by prolonging ventricular, systole reduce diastolic filling time. This latter effect, in the absence of effective atrial systole, will cause inefficient filling at faster rates.

We have seen that the position of the electrode within the right ventricle has some influence on its effect in lowering the aortic pressure. For any given rate the depression is greatest with apical stimulation and a wide QRS complex, and least with septal stimulation and a cardiogram showing minimal conduction aberration.

Our studies on conscious patients have shown that pacing rates exceeding 200 a minute are neces- 


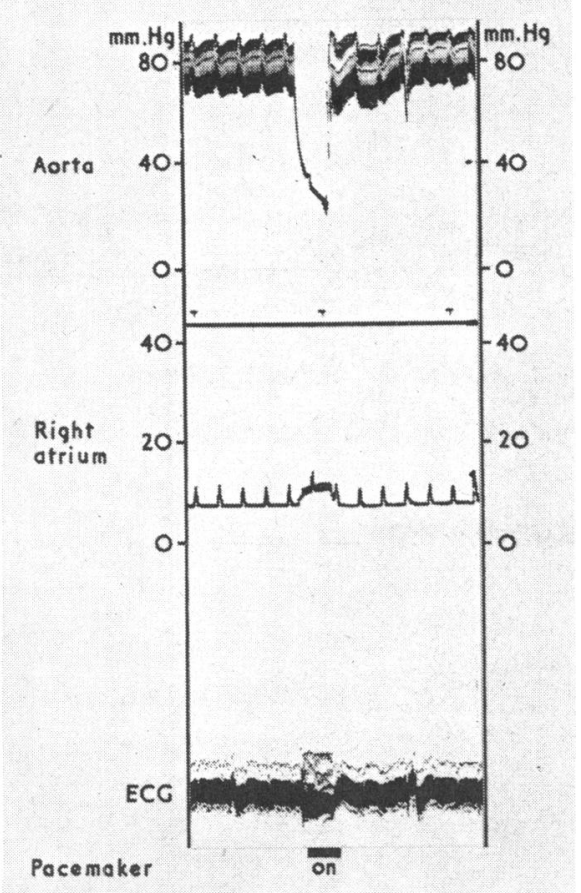

FIG. 4.-Simultaneous fall in aortic and rise in central venous pressure during rapid pacing.

sary to produce a significant fall in the aortic pressure, even after the administration of propranolol. In anaesthetized patients the degree of hypothermia down to $32^{\circ} \mathrm{C}$. has not proved important. In these patients the addition of ganglion blocking agents and propranolol will greatly enhance the effect of the pacemaker; nevertheless, despite the use of both agents, a rise in central venous pressure, invariably accompanying and following a period of circulatory arrest, will reduce the sensitivity of the cardiovascular system to the pacemaker, and necessitate a progressive increase in the rate of the pacing stimulus to maintain a low level of cardiac output.

It appears, therefore, that the extreme sensitivity of the blood pressure to right ventricular endocardial pacing is due to reduced efficiency of cardiac function from the abnormal rhythm and the failure of the vasomotor centre to compensate adequately by an increase in vascular motor tone.

When circulatory arrest has been established by balloon occlusion of the ascending aorta, the distal aortic and central venous pressures equilibrate at $5-15 \mathrm{~mm}$. $\mathrm{Hg}$ above the pre-arrest venous pressure. This value, the mean systemic pressure, is determined by blood volume and vascular tone, and agonal stimulation of the vasomotor reflexes causes it to rise progressively during the period of arrest (Fig. 3). This diminishes the efficiency of the pacemaker depression of cardiac function, and a progressive rise in pulmonary vascular pressure occurs, presumably due to shifts of blood from systemic to pulmonary circuits. These changes will take place more rapidly if the pacing stimulus is stopped immediately arrest has been established; at first left ventricular stroke volume is small, capable of maintaining only a moderate systolic pressure in the occluded ascending aorta, but the rising central venous and pulmonary venous pressures quickly reprime both ventricles, and within one minute there is an exponential rise in pulmonary arterial

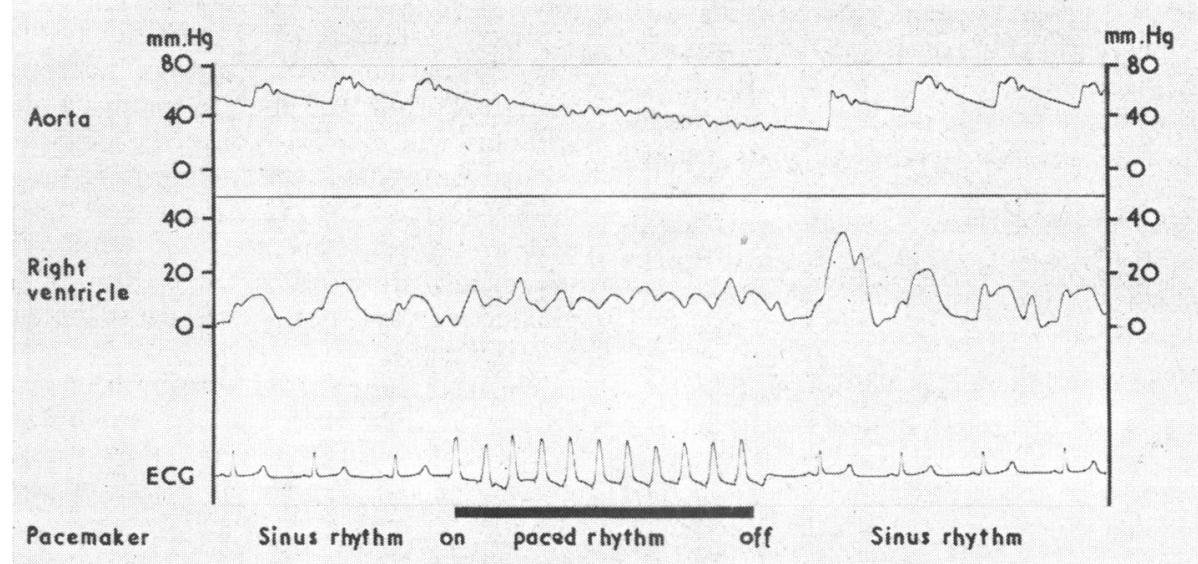

FIG. 5.-Increase in right ventricular end-diastolic pressure during pacing. First sinus beat following shows increased atrial and right ventricular systolic pressure and increased aortic pulse pressure. 


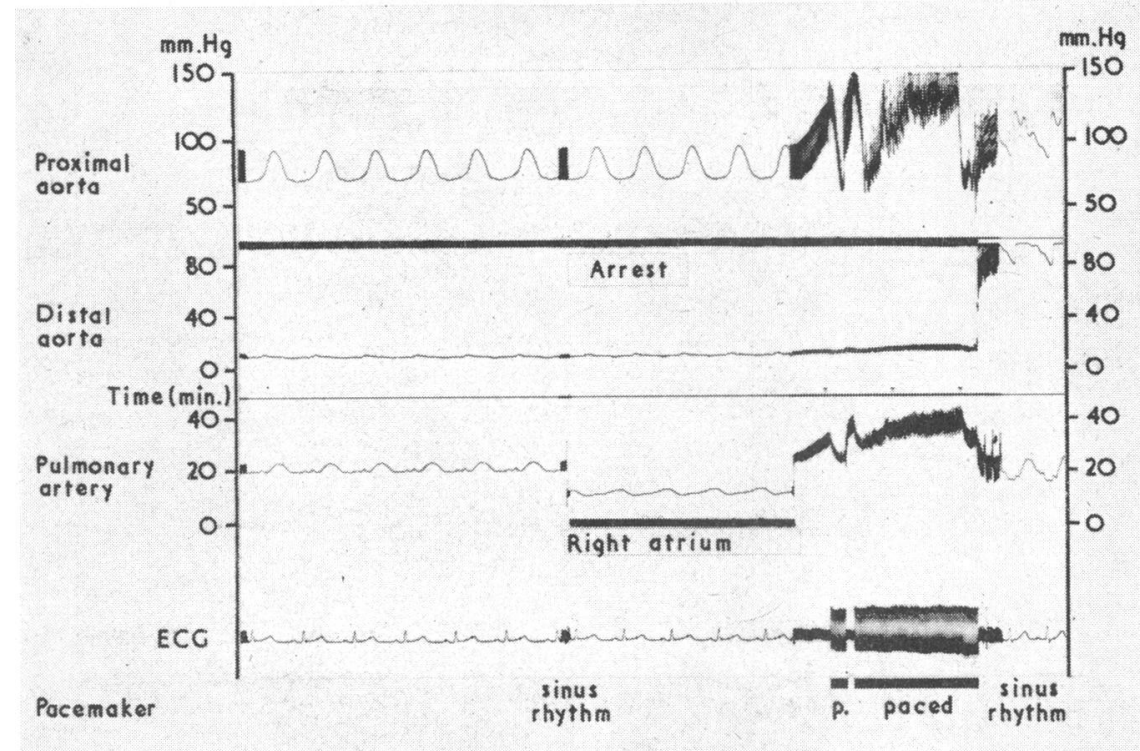

FIG. 6.-The pacemaker has been switched off during the first 60 seconds of the arrest and there is a rapid rise in proximal aortic and pulmonary arterial pressures necessitating further pacing. The pulmonary artery pressure falls to normal immediately on re-establishing the general circulation.

and aortic pressures. If the pacemaker is then reapplied at a rapid rate there is an immediate fall in pulmonary arterial pressure, followed shortly by a fall in ascending aortic pressure (Fig. 6).

The pacing rate necessary to maintain the occluded aortic pressure at $50-100 \mathrm{~mm}$. $\mathrm{Hg}$ depends upon the pre-arrest circulatory state: hypothermia, halothane anaesthesia, propranolol, and phenactropinium chloride provide the ideal pre-arrest state, allowing the balloon to be distended with an initial arrest stimulus of 120 a minute, and a satisfactory coronary perfusion pressure with complete aortic occlusion at an initial rate of 80 to 90 a minute rising to $120-130$ a minute for an arrest of 3 to 4 minutes (Fig. 7).

Progressive rise in pulmonary vascular pressure is an inevitable consequence of the method unless the pacing stimulus rate is increased sufficiently to balance the rise in mean systemic pressure, and this proved impossible in practice so that a certain amount of "hunting" in the ascending aortic pressure is inevitable. Any tendency for an excessive rise in aortic or pulmonary pressures can always be reversed by a further increase in pacing rate.

Measurement of actual coronary blood flow under these conditions is impossible, but, since this takes place mainly in diastole, resistance to flow must rise progressively with increased rates of stimulus. Nevertheless, with few exceptions, coronary flows have been sufficient to maintain the heart under control of the pacemaking stimulus during the arrest.

Metabolic acidosis is an inevitable consequence of circulatory arrest, and if this is uncorrected it will cause rhythmic disturbances such as bradycardia, heart block, and asystole during the immediate postarrest period, when the blood, which has lain in the systemic capillaries for several minutes, is washed into the heart and lungs by re-establishment of the circulation. Fig. 8A and 8B show sequential readings of the main parameters of blood gases and biochemistry during and immediately after an arrest. The venous blood taken from the right atrium shows much greater disturbance than that taken from the pulmonary artery, since it is not diluted with blood which has been passing through the coronary circulation and the lungs during the arrest period. The sharp rise in blood sodium and bicarbonate are due to sodium bicarbonate infusion.

Agonal discharge of adrenaline and noradrenaline provoke tachycardia, rebound hypertension, and dysrhythmia during the immediate post-arrest period. Intravenous propranolol and continued pacing at suitable rates will control these untoward effects.

\section{Complications}

Loss of Control of Heart Rhythm. This is the commonest and least serious complication. Postarrest atrial fibrillation occurred in about 20 per 


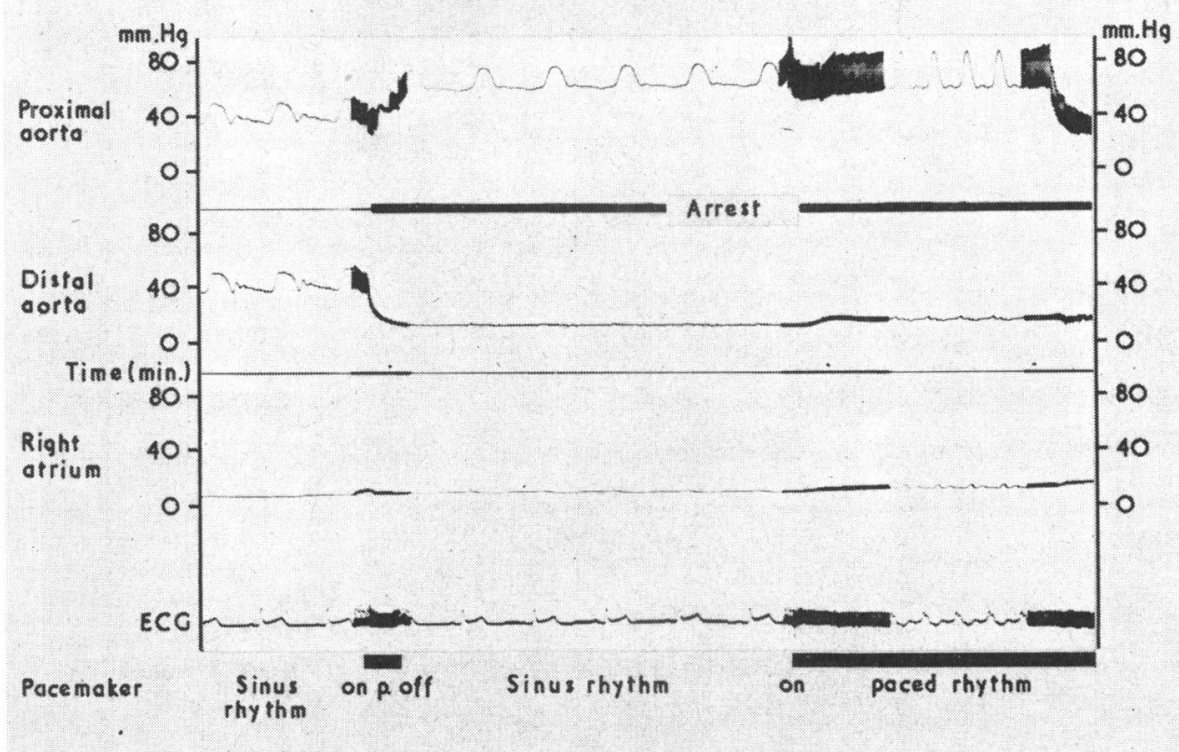

Fig. 7.-Hypotension induced before the arrest enables this to be maintained at relatively slow pacing rates.

cent of patients, and usually reverted spontaneously. Spontaneous ventricular fibrillation occurred on 6 occasions in 4 patients usually during the critical initial phase of arrest when the coronary perfusion pressure was low; this complication was avoided by rapid inflation of the balloon. When it occurred, immediate DC shock was given without prior cardiac massage and the arrest continued without further delay. This was successful in all cases but one.

A 19-year-old youth developed spontaneous ventricular fibrillation after approximately 3 minutes of arrest, with an ascending aortic pressure of $90 / 40 \mathrm{~mm} . \mathrm{Hg}$ and a pacing rate of approximately 100 a minute. Ten external DC shocks with intervals of external massage failed to revert the rhythm, and the chest was opened and cardiac massage continued, with 6 further shocks applied directly to the heart without success. The heart was then douched with warm saline and reverted to normal rhythm with the seventh shock, with immediate restoration of a normal circulation and blood pressure. There was an uneventful recovery despite a total arrest lasting 32 minutes. The pre-arrest temperature was $31^{\circ} \mathrm{C}$. and had been maintained at this level for several hours. The onset of the ventricular fibrillation and its resistance to shock were attributed to excessive hypothermia.

Thromboembolism. Two patients were thought to have suffered cerebral thromboembolism as a direct consequence of the procedure. Three patients developed transient symptoms and signs of ischaemia in the distribution of the right femoral artery, and one of these had mild persistent intermittent claudication; these complications occurred in the earlier cases and were eliminated by scrupulous removal of all clot from the femoral artery before suture.

The risk of thromboembolism remains, because there is a mandatory proscription on the use of heparin during neurosurgical operations, and because the technique requires a balloon catheter in the ascending aorta for several hours. Introduction of the catheter after body cooling has reduced this time by two hours, and if aortic catheterization and craniotomy proceeded together, using a portable fluoroscope with closed circuit television for positioning of the catheter, a further reduction would be possible. The newer type of silicone rubber for balloon construction would be an additional advantage.

Post-arrest Hypotension. A progressive fall in systemic arterial pressure after circulatory arrest occurred in 2 patients. One of these was known to be suffering from coronary arterial disease, and the hypotension was accompanied by significant shifts of the S-T segments in the electrocardiogram. In both instances the condition was corrected by partial inflation of the balloon to produce incomplete occlusion of the aorta. Observations with the fluoroscope have shown that this causes the balloon to be forced backwards into the descending aorta, 
$\underline{A}$
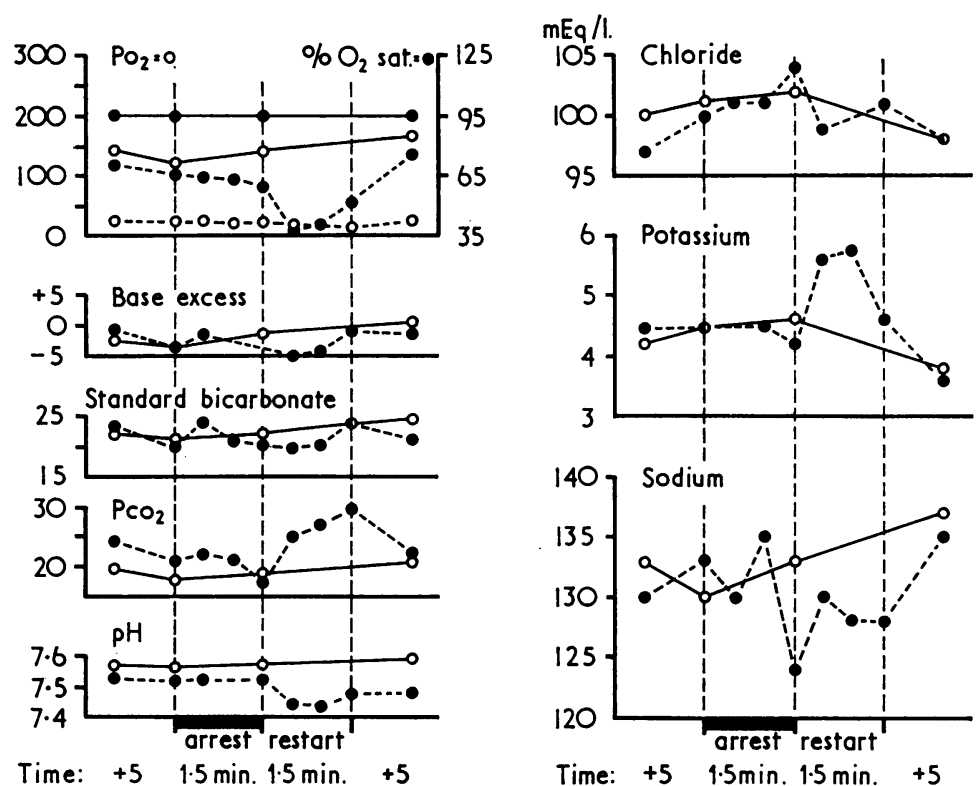

Time: $+5 \quad 1.5 \mathrm{~min} .1 .5 \mathrm{~min} . \quad+5$

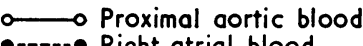

$\bullet----\bullet$ Right atrial blood

$\underline{B}$
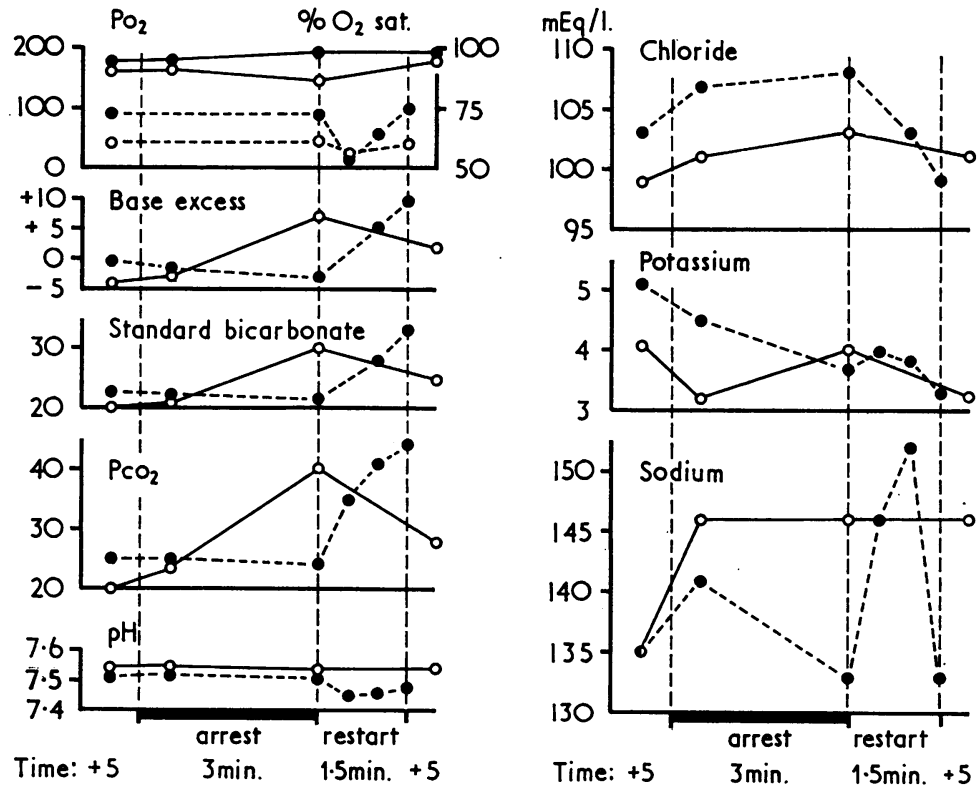

$\longrightarrow$ Proximal aortic blood

-.-.-. Pulmonary arterial blood

Fig. 8.-Biochemical changes in the blood during the arrest period (A) from aorta and right atrium, (B) from aorta and pulmonary artery. 


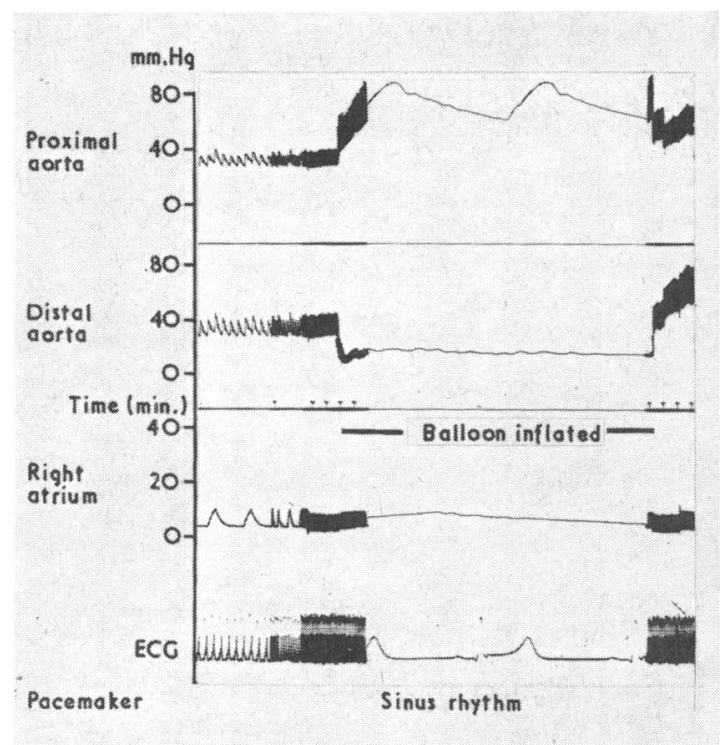

FIG. 9.-The effect of simple balloon inflation on postcirculatory arrest hypotension. thus provoking an artificial coarctation of the aorta and directing the greater part of the cardiac output to heart and head. An increase of the ascending aortic pressure in this way for several minutes corrected the hypotension which did not recur when the balloon was deflated (Fig. 9).

\section{SUMMARY}

Elective circulatory arrest using an intracardiac pacemaking electrode and occluding balloon in the ascending aorta has been used successfully in $\mathbf{3 0}$ patients, allowing circulatory arrest to be induced at will and maintained for periods of up to 6 minutes without loss of control of the cardiac rhythm.

We wish to acknowledge with grateful thanks generous grants from the British Heart Foundation and the Birmingham Regional Hospital Board for the purchase of equipment.

\section{REFERENCE}

Small, J. M., Stephenson, S. C. F., Campkin, T. V., Davison, P. H., and Mcllveen, D. J. S. (1966). Elective circulatory arrest by artificial pacemaker. Lancet, 1, 570 . 